\title{
Two-color Thomson scattering at FLASH
}

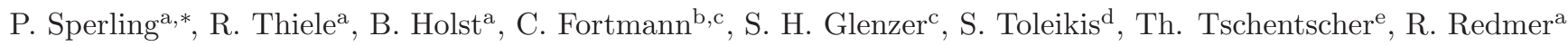 \\ ${ }^{a}$ Institut für Physik, Universität Rostock, D-18051 Rostock, Germany \\ ${ }^{b}$ Department of Physics and Astronomy, University of California-Los Angeles, Los Angeles, California 90095, USA \\ ${ }^{c}$ Lawrence Livermore National Laboratory, P.O. Box 808, L-399, Livermore, California 94551, USA \\ ${ }^{d}$ DESY, Notkestraße 85, D-22607 Hamburg, Germany \\ ${ }^{e}$ European XFEL GmbH, Albert-Einstein-Ring 19, D-22761 Hamburg, Germany
}

\begin{abstract}
We propose a two-color pump-probe Thomson scattering experiment at the FLASH facility in Hamburg to characterize warm dense matter states. The fundamental free electron laser wavelength of $40.5 \mathrm{~nm}$ is used to pump a liquid hydrogen jet that is subsequently probed with the third harmonic at $13.5 \mathrm{~nm}$. We have considered the laser-target interaction in the pump and probe phase by using the radiation hydrodynamics code HELIOS. The calculation of the Thomson scattering spectrum is based on the Chihara formula which is evaluated using the Born- Mermin approximation for the free electron dynamic structure factor and the Debye-Hückel static structure factor for the elastic scattering part. We consider the full density- and temperature-dependent Thomson scattering cross section throughout the inhomogeneous target. The results indicate that the electron-ion equilibration rate can be extracted by measuring the electron and ion feature with varying time delays between the pump and the probe pulse.
\end{abstract}

\section{Introduction}

X-ray Thomson scattering is a promising tool for the diagnostics of dense strongly correlated plasmas [1]. Xrays emitted from laser produced plasmas 2, 3] can probe the warm dense matter (WDM) region [4, 5] with temperatures of several $\mathrm{eV}$ and densities close to solid density [6, 7, 8] up to compressed matter well above solid density at electron temperatures $T_{e}$ between $0.1 \mathrm{eV}$ and several $10 \mathrm{eV}$ [9, 10, 11, 12, 13]. The study of dense plasmas is also possible with the implementation of free-electron lasers which provide brilliant radiation in the soft x-ray region at the free-electron LASer Hamburg (FLASH) at DESY, Hamburg [14, 15] or in the x-ray region at the Linac Coherent Light Source (LCLS) in Stanford [16], and will be available in the future at the European XFEL in Hamburg [17].

Collective x-ray Thomson scattering experiments yield information on the density and temperature of dense plasmas [8]. Assuming a homogeneous density and temperature throughout the target, these parameters can be determined directly from the plasmon dispersion and the ratio of the plasmon amplitudes via the detailed balance relation 18. For higher laser wavelengths, i.e., optical lasers and wavelengths of about $40-50 \mathrm{~nm}$ accessible with FLASH, the target is overdense, as the absorption is limited to the skin depth, in contrast to excitation by shortwavelength FEL radiation where gradients are smaller,

\footnotetext{
* Corresponding author

Email address: philipp.sperling@uni-rostock.de (P. Sperling)
}

see [19, 20]. The scattering signal represents an average of the local density- and temperature- dependent scattering cross-sections weighted with the respective density and temperature profiles [21, 22, 23].

In this paper, we propose a new Thomson scattering experiment at FLASH using a two-color pump-probe scheme on liquid hydrogen based on theoretical results obtained recently for an optical pump - FLASH probe setup 23]. This proposed experiment uses the FLASH fundamental as pump and the third harmonic as probe. We calculate the Thomson scattering spectrum of free electrons via the Born-Mermin approximation (BMA) 18, 24]. Furthermore, we determine the elastic scattering signal of bound electrons, the so-called Rayleigh signal, within the simple Debye-Hückel approximation. We find that a realistic description of the light-matter interaction within the proposed pump-probe experiment is of paramount importance, especially to derive reliable density and temperature profiles throughout the inhomogeneous target. We use the radiation hydrodynamics code HELIOS for the characterization of the target [25]. Our results indicate that the full time- resolved Thomson scattering spectrum can be used to extract electron-ion equilibration rates by varying the time delay between pump and probe pulse.

\section{Thomson scattering spectrum}

The experimentally available scattering power per solid angle $\mathrm{d} \Omega=\sin \vartheta \mathrm{d} \vartheta \mathrm{d} \varphi$ and per unit frequency interval $\mathrm{d} \omega$ 
is given by the following expression [21]:

$$
\begin{aligned}
\frac{\mathrm{d}^{2} P_{s c}}{\mathrm{~d} \Omega \mathrm{d} \omega} & =\frac{\sigma_{T}}{A_{\text {rad }}} \frac{k_{f}}{k_{i}} \int_{-\infty}^{\infty} \frac{\mathrm{d} \omega^{\prime}}{2 \pi} G_{\Delta \omega}\left(\omega-\omega^{\prime}\right) \\
& \times \int \mathrm{d}^{3} \mathbf{r} l(\mathbf{r}) S_{e e}\left(\mathbf{k}, \omega^{\prime} ; n_{e}(\mathbf{r}), T_{e}(\mathbf{r})\right) n_{i}(\mathbf{r}),
\end{aligned}
$$

where $\sigma_{T}=6.65 \times 10^{-24} \mathrm{~cm}^{2}$ is the Thomson scattering cross- section. The energy and momentum transfer are given by $\Delta E=\hbar \omega=\hbar \omega_{f}-\hbar \omega_{i}$ and $\hbar \mathbf{k}=\hbar \mathbf{k}_{f}-\hbar \mathbf{k}_{i}$, respectively. Here $k_{i}$ and $k_{f}$ are the initial and final photon wave numbers, and $\omega_{i}$ and $\omega_{f}$ are the initial and final photon frequencies. The momentum is related to the scattering angle $\theta$ in the limit $\hbar \omega \ll \hbar \omega_{0}$ according to $k=4 \pi \sin (\theta / 2) / \lambda_{0}$, with $\lambda_{0}$ being the probe wavelength. $l(\mathbf{r})$ is the $\mathbf{r}$-dependent power density of incoming photons which is also dependent on the absorption, $n_{i}(\mathbf{r})$ is the ion density, $n_{e}(\mathbf{r})$ is the electron density, and $A_{\text {rad }}$ is the irradiated surface of the target. The dynamic structure factor $S_{e e}(k, \omega)$ is convoluted with the instrumental function $G_{\Delta \omega}(\omega)$ that models the spectrometer's finite spectral resolution as well as the probe's spectral bandwidth. Usually, a normalized Gaussian distribution is employed with the full width at half maximum (FWHM) $\Delta \omega$, see also Ref. 22]. The dynamic structure factor can be separate into contributions of free electrons, weakly- and tightlybound electrons, and core electrons as proposed by Chihara [26, 27]:

$$
\begin{aligned}
S_{e e}(k, \omega) & =Z_{f} S_{e e}^{0}(k, \omega)+\left|f_{i}(k)+q(k)\right|^{2} S_{i i}(k, \omega) \\
& +Z_{c} \int_{-\infty}^{\infty} \mathrm{d} \omega^{\prime} S_{c}(k, \omega) S_{s}\left(k, \omega-\omega^{\prime}\right) .
\end{aligned}
$$

Here, $Z_{f}=n_{e} / n_{i}$ is the ionization degree of the plasma and $Z_{c}$ is the averaged number of core electrons. The first contribution of free electrons is calculated via the BMA which contains ion-electron collisions in second order Born approximation. For the calculation of the second term, we use the atomic form factor $f_{i}(k)$ [28] and the Debye-Hückel ion-ion structure factor for point charges

$$
S_{i i}(k)=\frac{k^{2}+\kappa_{e}^{2}}{k^{2}+\kappa_{i}^{2}+\kappa_{e}^{2}},
$$

with the inverse screening length $\kappa_{c}=\sqrt{e_{c}^{2} n_{c} /\left(\epsilon_{0} k_{\mathrm{B}} T_{c}\right)}$ for species $c=e$ (electrons) and $c=i$ (ions). In the Debye-Hückel picture, the screening cloud can be given by $q(k)=\sqrt{Z_{f}} S_{e i}(k) / S_{i i}(k)=Z_{f} \kappa_{e}^{2} /\left(k^{2}+\kappa_{e}^{2}\right)$. For the last term in Eq. (2), the contribution of bound-free transitions, we use the formalism of Schumacher et al. [28, 29]. This part describes the Raman-type transitions of inner shell electrons to the continuum which can be modulated by the ion's movement contained in $S_{s}(k, \omega)$ [30]. In this paper for Thomson scattering on hydrogen, the last term can be neglected for the relevant energy range of $-10 \mathrm{eV}$ to $10 \mathrm{eV}$.

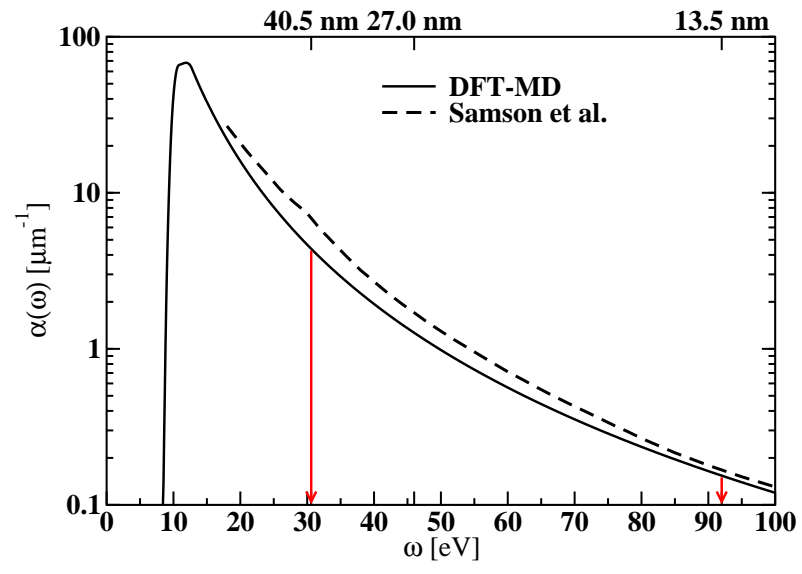

Figure 1: $A b$ initio simulation of the inverse absorption length $\alpha(\omega)$ for cryogenic hydrogen $(T=20 \mathrm{~K})$ at a liquid density of $\rho=0.086 \mathrm{~g} / \mathrm{cm}^{3}$ as function of the photon energy in comparison with Samson et al. [36]. The two red arrows indicates the fundamental wavelength of $40.5 \mathrm{~nm}$ and the $3 \mathrm{rd}$ harmonic at $13.5 \mathrm{~nm}$.

\section{Temperature and density profiles with HELIOS}

We describe the new experimental setup for Thomson scattering at FLASH briefly. We propose to use a fundamental wavelength of $40.5 \mathrm{~nm}(E=30.6 \mathrm{eV})$ for the pump pulse that generates an inhomogeneous plasma in the cryogenic hydrogen droplets of $30 \mu \mathrm{m}$ diameter, which has an initial state $T_{i}=20 \mathrm{~K}, \rho=0.086 \mathrm{~g} / \mathrm{cm}^{3}$. The $3 \mathrm{rd}$ harmonic with a wavelength of $13.5 \mathrm{~nm}(E=92 \mathrm{eV})$ is applied as probe pulse which undergoes Thomson scattering processes in the target. The fundamental and the third harmonic have each a pulse duration of $150 \mathrm{fs}$ and a focal spot radius of $20 \mu \mathrm{m}$. The pulse energy of the fundamental is $250 \mu \mathrm{J}$ and that of the third harmonic is $2.5 \mu \mathrm{J}$.

For a more detailed description of the absorption process, we calculate the inverse absorption length $\alpha(\omega)=$ $1 / l_{a b s}(\omega)$ of cryogenic hydrogen via ab initio molecular dynamic simulations using the VASP code [31, 32, 33. By applying the projector- augmented wave method [34], we are able to evaluate the Kubo- Greenwood formula to obtain optical properties 35], which are compared with experimental data from Samson et al. 36, see Fig. 1 The absorption length for $40.5 \mathrm{~nm}$ (pump) is about thirty times shorter than for $13.5 \mathrm{~nm}$ (probe) so that the energy of the pump pulse is deposited in a layer of only few $\mu \mathrm{m}$ thickness in the front of the droplet. This plasma region may yield a clear plasmon scattering signal when probed with the shorter wavelength of $13.5 \mathrm{~nm}$, similar to the results given in Ref. 23.

In Fig. 2 and Fig. 3, we show the results for the freeelectron density and the temperature profiles for the middle layer of the hydrogen droplet using the radiation-hydrodynamic simulation code HELIOS 25]. HELIOS features a Lagrangian reference frame, separate ion and electron temperatures, and flux-limited Spitzer thermal conductivity. The laser energy is deposited via inverse bremsstrahlung as well as bound-bound and bound-free transitions using 


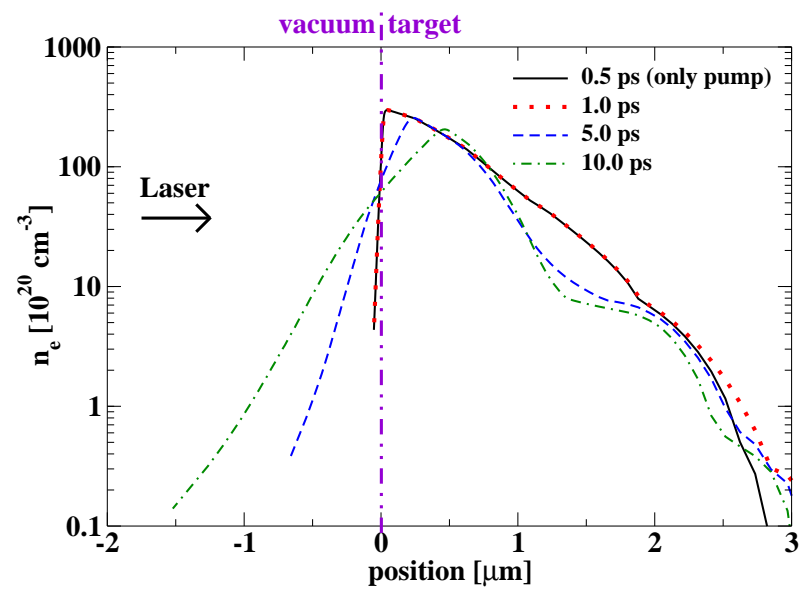

Figure 2: Free-electron density profiles $n_{e}(r)$ for different delay times between pump and probe pulse as derived from the HELIOS simulations. The black curve is the result for the plasma conditions $0.5 \mathrm{ps}$ after the pump pulse without irradiation by a probe pulse. Both FLASH laser pulses (pump: $40.5 \mathrm{~nm}$, probe: $13.5 \mathrm{~nm}, 150 \mathrm{fs}$ pulse length each) come from the left.

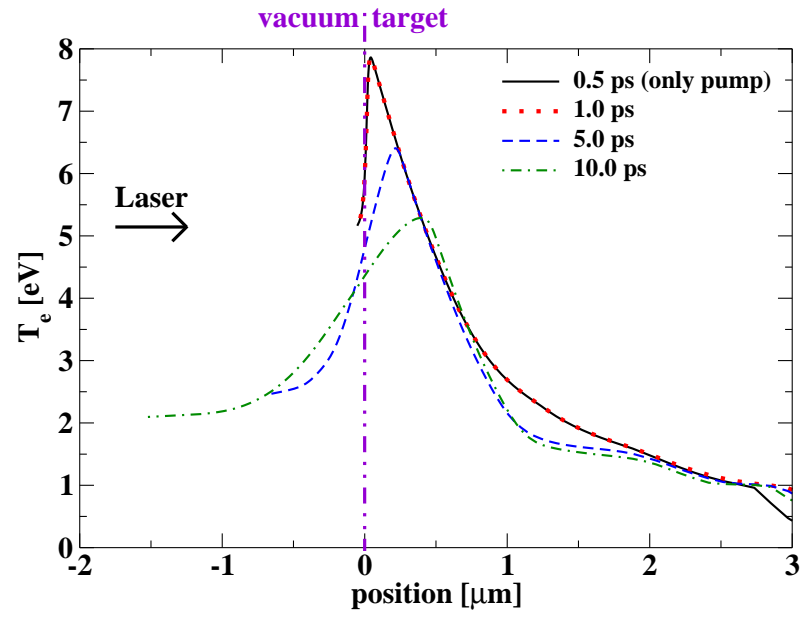

Figure 3: Temperature profile of free electrons $T_{e}(r)$ as derived from the HELIOS simulations, see Fig. 2

a SESAME-like equation of state 37. We show results for several delay times between pump and probe beam. The black curve with $t=0.5 \mathrm{ps}$ is the result for a target without irradiation by a probe pulse. With increasing delay times a progressing expansion of the plasma occurs and a shock front penetrates into the target. The maximum of the temperature curves is broadened with increasing delay time because of thermal flux.

In Fig. 2 and Fig. 3 a higher absorption is seen due to the higher wavelength of $40.5 \mathrm{~nm}$ in comparison with previous results of Fortmann et al. 22] where a wavelength of $13.5 \mathrm{~nm}$ was considered for self-Thomson scattering. Therefore, the first few $\mu \mathrm{m}$ of the target are well ionized and a subsequent hydrodynamic expansion is caused by the laser heating. In the remaining part of the target, the plasma temperature and density is very low due to the low penetration depth of the pump laser.

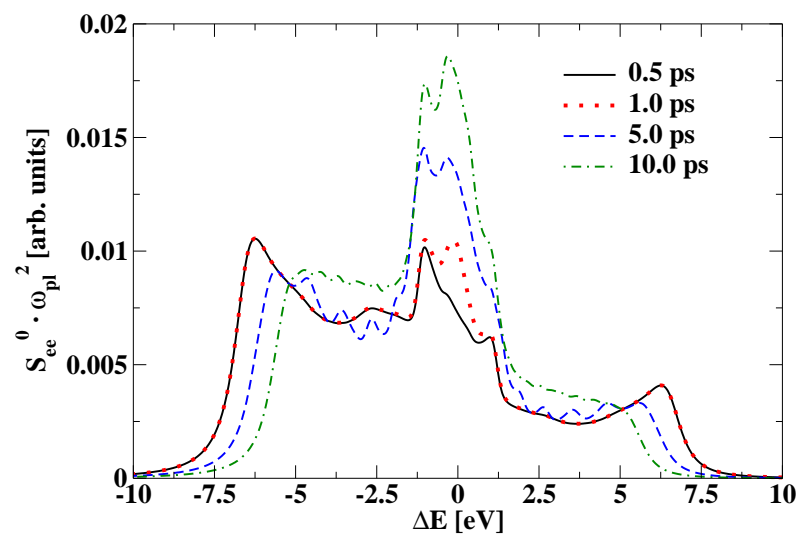

Figure 4: Free-electron Thomson scattering spectrum from liquid hydrogen droplets as function of the photon energy shift $\Delta E$. The "ideal" probe (black curve) is compared with the full pump-probe scenario considering delays of 1 ps (dotted red line), 5.0 ps (broken blue line), and 10 ps (broken dotted dark green line).

\section{Results for the Thomson scattering spectrum}

Using the electron density and temperature profiles through the hydrogen droplet as derived from the HELIOS simulations as input in Eq. (1), we calculate the Thomson scattering spectrum of the probe pulse for several delay times. Non-convoluted Thomson scattering spectra are shown in Fig. 4. Please note that the curves in Fig. 4 show only the contribution of free electrons. The HELIOS one-dimensional free-electron densities and temperatures are divided in 450 equidistant sampling points. For each cell we have calculated the dynamic structure factor $S_{e e}^{0}(k, \omega) \omega_{p e}^{2}$ with the electronic plasma frequency $\omega_{p e}^{2}=$ $n_{e} e^{2} /\left(\epsilon_{0} m_{e}\right)$ for a scattering angle of $\theta_{S}=90^{\circ}$. For a full scattering spectrum, all cells are weighted by the factor $\exp \left(-r / l_{a b s}\right)$, with the absorption length of $l_{a b s}=11 \mu \mathrm{m}$ as derived from Fig. 1 for $\lambda_{0}=13.5 \mathrm{~nm}$. Therefore, we consider the weakening of the incoming $13.5 \mathrm{~nm}$ FLASH probe pulse with increasing target depth.

The high plasma density in the outer shell of the droplets yields a strong plasmon peak in the spectrum at an energy shift of about $\Delta E=6.25 \mathrm{eV}$ for a 1 ps delay time. Later, the densities decrease and the plasmon peak is shifted to lower energies. For the maximum delay time of 10 ps considered, the plasmon peaks have already disappeared. The central peak in the spectrum can be related to collective as well as non-collective scattering from the lower density region in the droplet behind the shock front.

An important question for such Thomson scattering experiments is whether or not the plasmon peaks shown in Fig. 4 can be distinguished from the central Rayleigh peak which measures the elastic scattering from bound electrons. Therefore, and in extension of previous theoretical work [23], we have also calculated the respective second term in Eq. (2). For simplicity we apply the DebyeHückel approximation which is sufficient for our purposes here. Improved treatments, e.g., by performing hypernetted chain calculations (HNC) or ab initio molecular dy- 


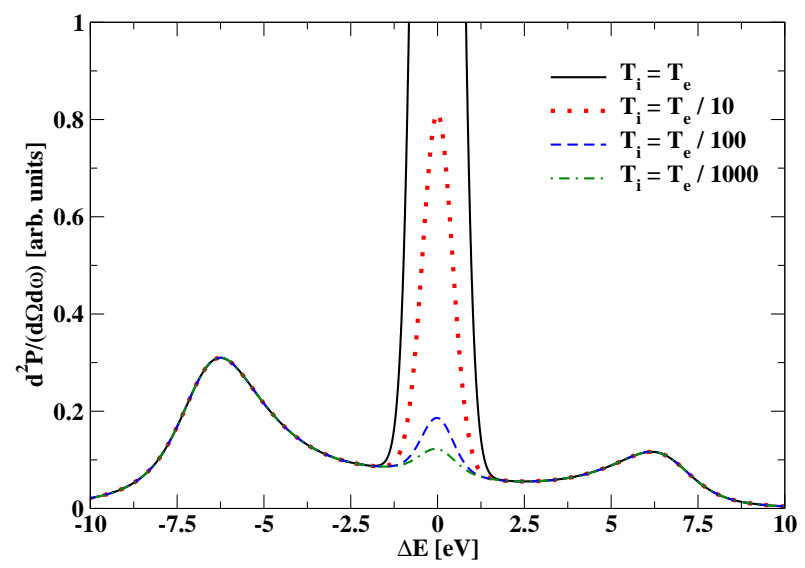

Figure 5: Convoluted full Thomson scattering spectrum from liquid hydrogen droplets as function of the photon energy shift $\Delta E$ for a delay time of $1 \mathrm{ps}$ and various atomic temperatures $T_{a}$. The electron temperatures $T_{e}$ are obtained from the HELIOS simulations. The Rayleigh peak increases with atomic temperature. For the convolution, a normalized Gaussian distribution with a FWHM of $\Delta \omega=1 \mathrm{eV}$ is employed.

namics simulations for the static ion-ion structure factor $S_{i i}(k)$, are straightforward [38, 39, 40].

As an example we show in Fig. 5 the total Thomson scattering spectrum including the ion feature for a delay time of $1 \mathrm{ps}$ and different temperatures of the atoms $T_{a}$. The ion feature represents the contribution of bound electrons which in the case of hydrogen refer to atoms. We assume that the atom temperature is equal to the temperature of the ions $T_{i}=T_{a}$. For a more detailed treatment of a three-component, partially ionized system with different temperatures $T_{e}, T_{i}$, and $T_{a}$, see Ref. [40, 41]. The spectra are convoluted with a FWHM $\Delta \omega=1 \mathrm{eV}$. For a fully ionized plasma, the Rayleigh peak would be zero. In case of partial ionization, especially for equilibrium with $T_{a}=T_{e}$, the Rayleigh peak stemming from the weakly- and tightly-bound electrons is a factor of about 30 times higher than the plasmon peak. Furthermore, the calculations show that the Rayleigh peak increases with the temperature of the atoms. Since a non-equilibrium state with $T_{a} \ll T_{e}$ is relevant for short times of a few ps after the pump pulse, the equilibration rate between the free electrons and the heavy particles (ions, atoms) can be derived by measuring the heights of the plasmon and Rayleigh peaks simultaneously. This is in principle possible via the proposed two-color pump- probe experiments at the FLASH facility.

\section{Conclusion}

We have shown with detailed calculations and modeling that electron-ion temperature equilibration in warm dense hydrogen can be measured with two-color Thomson scattering experiments at the FLASH facility. The proposed setup would use a fundamental wavelength of $40.5 \mathrm{~nm}$ to pump liquid hydrogen droplets and the third harmonic at $13.5 \mathrm{~nm}$ to probe the inhomogeneous plasma. Radiation- hydrodynamics simulations using the HELIOS code determined the expected electron density and temperature profiles in the droplet. The pump pulse induces strong gradients in the free electron density and temperature. The Thomson scattering spectrum is calculated via the Born-Mermin approximation for the electron feature and the Debye-Hückel structure factor for the ion feature. The high- density plasma in a thin surface layer of the droplet generates a plasmon signal which could be observed for short delay times of less than 5 ps. The Rayleigh signal of tightly-bound electrons is very large compared to the plasmon signal of free electrons for equilibrium conditions, i.e., $T_{a}=T_{e}$. For short times of few ps, we expect a non-equilibrium plasma with substantially lower temperatures for the heavy particles (ions, atoms), i.e., $T_{a} \ll T_{e}$, and thus a much smaller Rayleigh signal. Therefore, the equilibration rate between the free electron system and the heavy particles could be derived by measuring the heights of the plasmon and Rayleigh peaks simultaneously via the proposed two-color pump-probe experiments at FLASH. The evaluation of the scattering signal would also require more realistic ion-ion structure factors for the ion feature than the simple Debye-Hückel expression used here. The performance of $\mathrm{HNC}$ or ab initio MD simulations can be checked via k-dependent measurements of the Rayleigh signal using different scattering angles. Since multi-angle spectrometers are currently being designed, see e.g., [42], this task seems to be feasible.

\section{Acknowledgments}

We thank Th. Bornath, E. Förster, G. Gregori, H. Reinholz, G. Röpke, and U. Zastrau for helpful discussions. This work was supported by the DFG within the SFB 652 "Strong correlations and collective effects in radiation fields Coulomb systems, clusters, and particles" and the Federal Ministry for Education and Science (BMBF) under Grant No. FSP 301-FLASH and Project No. 05KS7HRA. C.F. acknowledges support by the Alexander von HumboldtFoundation.

[1] S. H. Glenzer, R. Redmer, X-ray Thomson scattering in high energy density plasmas, Rev. Mod. Phys. 81 (2009) 1625.

[2] S. H. Glenzer, W. Rozmus, B. J. MacGowan, K. G. Estabrook, J. D. De Groot, G. B. Zimmerman, H. A. Baldis, J. A. Harte, R. W. Lee, E. A. Williams, B. G. Wilson, Thomson scattering from high- $z$ laser-produced plasmas, Phys. Rev. Lett. 82 (1999) 97.

[3] G. Gregori, S. H. Glenzer, H.-K. Chung, D. Froula, R. Lee, N. Meezan, J. Moody, C. Niemann, O. Landen, B. Holst, R. Redmer, S. Regan, H. Sawada, Measurement of carbon ionization balance in high-temperature plasma mixtures by temporally resolved x-ray scattering, J. Quant. Spectrosc. Radiat. Transfer 99 (2006) 225.

[4] R. Lee, H. Baldis, R. Cauble, O. Landen, J. Wark, A. Ng, S. Rose, C. Lewis, D. Riley, J.-C. Gauthier, P. Audebert, Plasma-based studies with intense $\mathrm{x}$-ray and particle beam sources, Laser Part. Beams 20 (2002) 527.

[5] R. W. Lee, S. J. Moon, H.-K. Chung, W. Rozmus, H. A. Baldis, G. Gregori, R. C. Cauble, O. L. Landen, J. S. Wark, A. Ng, S. J. 
Rose, C. L. Lewis, D. Riley, J.-C. Gauthier, P. Audebert, Finite temperature dense matter studies on next-generation light sources, J. Opt. Soc. Am. B 20 (2003) 770.

[6] S. H. Glenzer, G. Gregori, R. W. Lee, F. J. Rogers, S. W. Pollaine, O. L. Landen, Demonstration of spectrally resolved x-ray scattering in dense plasmas, Phys. Rev. Lett. 90 (2003) 175002.

[7] G. Gregori, S. H. Glenzer, W. Rozmus, R. W. Lee, O. L. Landen, Theoretical model of $\mathrm{x}$-ray scattering as a dense matter probe, Phys. Rev. E 67 (2003) 026412.

[8] S. H. Glenzer, O. L. Landen, P. Neumayer, R. W. Lee, K. Widmann, S. W. Pollaine, R. J. Wallace, G. Gregori, A. Höll, T. Bornath, R. Thiele, V. Schwarz, W.-D. Kraeft, R. Redmer, Observations of plasmons in warm dense matter, Phys. Rev. Lett. 98 (2007) 065002.

[9] S. H. Glenzer, P. Neumayer, T. Döppner, O. L. Landen, R. W. Lee, R. J. Wallace, S. Weber, H. J. Lee, A. L. Kritcher, R. Falcone, S. P. Regan, H. Sawada, D. D. Meyerhofer, G. Gregori, C. Fortmann, V. Schwarz, R. Redmer, Compton scattering measurements from dense plasmas, Journal of Physics: Conference Series 112 (2008) 032071.

[10] H. J. Lee, P. Neumayer, J. Castor, T. Döppner, R. W. Falcone, C. Fortmann, B. A. Hammel, A. L. Kritcher, O. L. Landen, R. W. Lee, D. D. Meyerhofer, D. H. Munro, R. Redmer, S. P. Regan, S. Weber, S. H. Glenzer, X-ray Thomson-scattering measurements of density and temperature in shock-compressed beryllium, Phys. Rev. Lett. 102 (2009) 115001.

[11] A. L. Kritcher, P. Neumayer, J. Castor, T. Döppner, R. W. Falcone, O. L. Landen, H. J. Lee, R. W. Lee, E. C. Morse, A. Ng, S. Pollaine, D. Price, S. H. Glenzer, Ultrafast x-ray Thomson scattering of shock-compressed matter, Science 322 (2008) 69.

[12] E. G. Saiz, G. Gregori, D. O. Gericke, J. Vorberger, B. Barbrel, R. J. Clarke, R. R. Freeman, S. H. Glenzer, F. Y. Khattak, M. Koenig, O. L. Landen, D. Neely, P. Neumayer, M. M. Notley, A. Pelka, D. Price, M. Roth, M. Schollmeier, C. Spindloe, R. L. Weber, L. van Woerkom, K. Wünsch, D. Riley, Probing warm dense lithium by inelastic x-ray scattering, Nat. Phys. 4 (2008) 940.

[13] S. Glenzer, H. Lee, P. Davis, T. Döppner, R. Falcone, C. Fortmann, B. Hammel, A. Kritcher, O. Landen, R. Lee, D. Munro, R. Redmer, S. Weber, Dense plasma x-ray scattering: Methods and applications, High Energy Dens. Phys. 6 (2010) 1.

[14] S. Toleikis, R. Fäustlin, L. Cao, T. Döppner, S. Düsterer, E. Förster, C. Fortmann, S. Glenzer, S. Göde, G. Gregori, R. Irsig, T. Laarmann, H. Lee, B. Li, J. Mithen, K.-H. MeiwesBroer, A. Przystawik, P. Radcliffe, R. Redmer, F. Tavella, R. Thiele, J. Tiggesbäumker, N. Truong, I. Uschmann, U. Zastrau, T. Tschentscher, Soft x-ray scattering using fel radiation for probing near-solid density plasmas at few electron volt temperatures, High Energy Dens. Phys. 6 (2010) 15.

[15] R. Fäustlin, T. Bornath, T. Döppner, S. Düsterer, E. Förster, C. Fortmann, S. H. Glenzer, S. Göde, G. Gregori, R. Irsig, T. Laarmann, H. Lee, B. Li, K.-H. Meiwes-Broer, J. Mithen, B. Nagler, A. Przystawik, H. Redlin, R. Redmer, H. Reinholz, G. Röpke, F. Tavella, R. Thiele, J. Tiggesbäumker, S. Toleikis, I. Uschmann, S. Vinko, T. Whitcher, U. Zastrau, B. Ziaja, T. Tschentscher, Observation of ultrafast non-equilibrium collective dynamics in warm dense hydrogen, Phys. Rev. Lett. 104 (2010) 125002.

[16] Y. Ding, A. Brachmann, F.-J. Decker, D. Dowell, P. Emma, J. Frisch, S. Gilevich, G. Hays, P. Hering, Z. Huang, R. Iverson, H. Loos, A. Miahnahri, H.-D. Nuhn, D. Ratner, J. Turner, J. Welch, W. White, J. Wu, Measurements and simulations of ultralow emittance and ultrashort electron beams in the linac coherent light source, Phys. Rev. Lett. 102 (2009) 254801.

[17] M. Altarelli, R. Brinkmann, M. Chergui, W. Decking, B. Dobson, S. Düsterer, G. Grübel, W. Graeff, H. Graafsma, J. Hajdu, J. Marangos, J. Pflüger, H. Redlin, D. Riley, I. Robinson, J. Rossbach, A. Schwarz, K. Tiedtke, T. Tschentscher, I. Vartaniants, H. Wabnitz, H. Weise, R. Wichmann, K. Witte, A. Wolf, M. Wulff, M. Yurkov (Eds.), The European X-Ray Free-Electron Laser - Technical design report, DESY 2006-097,
Hamburg, 2007

[18] A. Höll, T. Bornath, L. Cao, T. Döppner, S. Düsterer, E. Förster, C. Fortmann, S. H. Glenzer, G. Gregori, T. Laarmann, K.-H. Meiwes-Broer, A. Przystawik, P. Radcliffe, R. Redmer, H. Reinholz, G. Röpke, R. Thiele, J. Tiggesbäumker, J. Toleikis, N. X. Truong, T. Tschentscher, I. Uschmann, U. Zastrau, Thomson scattering from near-solid density plasmas using soft x-ray free electron lasers, High Energy Dens. Phys. 3 (2007) 120.

[19] U. Zastrau, C. Fortmann, R. R. Fäustlin, L. F. Cao, T. Döppner, S. Düsterer, S. H. Glenzer, G. Gregori, T. Laarmann, H. J. Lee, A. Przystawik, P. Radcliffe, H. Reinholz, G. Röpke, R. Thiele, J. Tiggesbäumker, N. X. Truong, S. Toleikis, I. Uschmann, A. Wierling, T. Tschentscher, E. Förster, R. Redmer, Bremsstrahlung and line spectroscopy of warm dense aluminum plasma heated by XUV free-electronlaser radiation, Phys. Rev E 78 (2008) 066406.

[20] B. Nagler, U. Zastrau, R. R. Fäustlin, S. M. Vinko, T. Whitcher, A. J. Nelson, R. Sobierajsk, J. Krzywinski, J. Chalupsky, E. Abreu, S. Bajt, T. Bornath, T. Burian, H. Chapman, J. Cihelka, T. Döppner, S. Düsterer, T. Dzelzainis, M. Fajardo, E. Förster, C. Fortmann, E. Galtier, S. H. Glenzer, S. Göde, G. Gregori, V. Hajkov, P. Heimann, L. Juha, M. Jurek, F. Y. Khattak, A. R. Khorsand, D. Klinger, M. Kozlova, T. Laarmann, H. J. Lee, R. Lee, K.-H. Meiwes-Broer, P. Mercere, W. J. Murphy, A. Przystawik, R. Redmer, H. Reinholz, D. Riley, G. Röpke, F. Rosmej, K. Saksl, R. Schott, R. Thiele, J. Tiggesbäumker, S. Toleikis, T. Tschentscher, I. Uschmann, H. J. Vollmer, J. S.Wark, Turning solid aluminium transparent by intense soft x-ray photoionization, Nat. Phys. 5 (2009) 693.

[21] H. A. Baldis, J. Dunn, M. E. Foord, W. Rozmus, Thomson scattering diagnostic of solid density plasmas using x-ray lasers, Rev. Sci. Instrum. 73 (2002) 4223.

[22] C. Fortmann, R. Thiele, R. Fäustlin, T. Bornath, B. Holst, W.D. Kraeft, V. Schwarz, S. Toleikis, T. Tschentscher, R. Redmer, Thomson scattering in dense plasmas with density and temperature gradients, High Energy Dens. Phys. 5 (2009) 208.

[23] R. Thiele, P. Sperling, M. Chen, T. Bornath, R. R. Fäustlin, C. Fortmann, S. H. Glenzer, W.-D. Kraeft, A. Pukhov, S. Toleikis, T. Tschentscher, R. Redmer, Thomson scattering on inhomogeneous targets, Phys. Rev. E 82 (2010) 056404.

[24] C. Fortmann, T. Bornath, R. Redmer, H. Reinholz, G. Röpke, V. Schwarz, R. Thiele, X-ray thomson scattering cross-section in strongly correlated plasmas, Laser Part. Beams 27 (2009) 311.

[25] J. MacFarlane, I. Golovkin, P. Woodruff, HELIOS-CR-A 1-D radiation-magnetohydrodynamics code with inline atomic kinetics modeling, J. Quant. Spectrosc. Radiat. Transfer 99 (2006) 381.

[26] J. Chihara, Difference in x-ray scattering between metallic and non-metallic liquids due to conduction electrons, J. Phys. F 17 (1987) 295.

[27] J. Chihara, Interaction of photons with plasmas and liquid metals - photoabsorption and scattering, J. Phys. Cond. Matter 12 (2000) 231.

[28] M. Schumacher, F. Smend, I. Borchert, Incoherent scattering of gamma rays by inner-shell electrons, Journal of Physics B: Atomic and Molecular Physics 8 (9) (1975) 1428.

[29] G. Gregori, S. H. Glenzer, F. J. Rogers, S. M. Pollaine, O. L. Landen, C. Blancard, G. Faussurier, P. Renaudin, S. Kuhlbrodt, R. Redmer, Electronic structure meassurements of dense plasmas, Phys. Plasmas 11 (2004) 2754.

[30] S. Sahoo, G. F. Gribakin, G. S. Naz, J. Kohanoff, D. Riley, Compton scatter profiles for warm dense matter, Phys. Rev. E 77 (2008) 046402.

[31] G. Kresse, J. Hafner, Abinitio molecular-dynamics for liquidmetals, Phys. Rev. B 47 (1993) 558.

[32] G. Kresse, J. Hafner, Ab-initio molecular-dynamics simulation of the liquid-metal amorphous-semiconductor transition in germanium, Phys. Rev. B 49 (1994) 14251.

[33] G. Kresse, J. Furthmüller, Efficient iterative schemes for ab ini- 
tio total-energy calculations using a plane-wave basis set, Phys. Rev. B 54 (1996) 11169.

[34] G. Kresse, D. Joubert, From ultrasoft pseudopotentials to the projector augmented-wave method, Phys. Rev. B 59 (1999) 1758.

[35] B. Holst, R. Redmer, M. P. Desjarlais, Thermophysical properties of warm dense hydrogen using quantum molecular dynamics simulations, Phys. Rev. B 77 (2008) 184201.

[36] J. A. R. Samson, G. N. Haddad, Total photoabsorption cross sections of $\mathrm{H}_{2}$ from 18 to $113 \mathrm{eV}$, J. Opt. Soc. Am. B 11 (1994) 277 .

[37] S. P. Lyon, J. D. Johnson, SESAME: The Los Alamos National Laboratory Equation of State Database, Tech. Rep. LA-UR92-3407, Los Alamos National Laboratory, Los Alamos, NM (1992).

[38] V. Schwarz, B. Holst, T. Bornath, C. Fortmann, W.-D. Kraeft, R. Thiele, R. Redmer, G. Gregori, H. J. Lee, T. Dppner, S. H. Glenzer, Static ion structure factor for dense plasmas: Semiclassical and ab initio calculations, High Energy Density Physics 6 (2010) 305.

[39] K. Wünsch, P. Hilse, M. Schlanges, D. O. Gericke, Structure of strongly coupled multicomponent plasmas, Phys. Rev. E 77 (2008) 056404.

[40] J. Vorberger, D. O. Gericke, T. Bornath, M. Schlanges, Energy relaxation in dense, strongly coupled two-temperature plasmas, Phys. Rev. E 81 (2010) 046404.

[41] T. Ohde, M. Bonitz, T. Bornath, D. Kremp, M. Schlanges, Twotemperature relaxation in nonideal partially ionized plasmas, Phys. Plasmas 3 (1996) 1241.

[42] R. R. Fäustlin, U. Zastrau, S. Toleikis, I. Uschmann, E. Förster, T. Tschentscher, A compact soft x-ray spectrograph combining high efficiency and resolution, Journal of Instrumentation 5 (2010) P02004. 